\title{
An Enhanced Multiple-Feedback Algorithm for RFID MAC Protocols
}

\author{
You-Chang $\mathrm{Ko}^{1}$, Sumit Roy ${ }^{2}$, Choong-Ho $\mathrm{Cho}^{3}$, and Hyong-Woo Lee ${ }^{4}$ \\ ${ }^{1}$ Solution Business Division, Samsung Networks Inc., Seoul, S. Korea \\ ${ }^{1}$ youchang.ko@samsung.com \\ ${ }^{2}$ Dept. of Electrical Engineering, University of Washington, Seattle, WA 98195 \\ sroy@u.washington.edu \\ ${ }^{3}$ Dept. of Computer \& Information Science, Korea University, Jochiwon, S. Korea \\ ${ }^{3}$ chcho@korea.ac.kr \\ ${ }^{4}$ Dept. of Electronics \& Information Engineering, Korea University, Jochiwon, S. Korea \\ ${ }^{4} \mathrm{hwlee@korea.ac.kr}$
}

\begin{abstract}
This paper introduces two new tree-based anticollision schemes using multiple feedback symbols for uplink tag random access of emerging radio frequency identification (RFID) networks. To this end a uplink(UL) time slot requires an extra time portion called voting field in addition to the packet payload to obtain a slot based estimate for the number of contending tags. As a function of the voting field length we examine MAC efficiency improvements in terms of UL throughput, and compare it with the conventional binary tree based RFID MAC in ISO 18000-6 Type B standard.
\end{abstract}

\section{INTRODUCTION}

The radio frequency identification (RFID) network itself has two principal components: a) the reader/interrogator and b) objects with attached tags/transponders. The primary metric for evaluation of an RFID network is rate of acquiring tag information by the reader upon querying. While physical layer air interface parameters such as data transmission rate in downlink(DL, reader-to-tag)/uplink(UL, tag-to-reader) clearly impact the tag read rate, the impact of the multiple access protocol (and specifically the anti-collision component) is the focus of this work. The great majority of the plethora of anticollision schemes in the RFID MAC arena that have been proposed thus far, have been categorized into two primary types - framed Aloha[7],[8] and m-ary splitting algorithm (MSA)[3]-[6],[9],[10], where both schemes are designed to broadcast a single feedback symbol for packet access result to a UL time slot. MSA can be further classified into two categories depending on the sequence of collision resolution process (CRP): depth-first-search MSA(DMSA)[3]-[6] and breadth-first-search MSA(BMSA)[9],[10]. In this paper we introduce a novel anti-collision principle called multiple feedback algorithm (MFA) based on [3], and propose its enhanced version (EMFA) into RFID MAC protocols. The (E)MFA running on top of the DMSA ${ }^{1}$ skeleton structure utilizes a novel approach in which the reader broadcasts

${ }^{1}[4]$-[6] are the special case of DMSA where $m=2$, a fixed binary tree MAC, whereas MFA[3] is the special case of DMSA where $m$ is dynamic per collided slot. multiple feedback symbols for a UL time slot. To this end a UL time slot requires an extra time portion called voting field in addition to the packet payload to obtain a slot based estimate for the number of contending tags. Fig. 1 illustrates the slot structure of the conventional schemes and the (E)MFA in a half duplex shared medium. Consider the $x$-th time slot $\left(S_{x}\right)$ in a TDMA uplink frame. Then the length of a UL time slot in conventional schemes $\left(T_{c_{-} s l t}\right)$ is set exactly the same as the packet duration $\left(T_{p k t}\right)$ for which a slot based single feedback - a ternary type random variable $(Q)$, i.e., Empty, Success or Collision - is implicitly or explicitly broadcast via downlink (DL) channel according to DMSA protocol as seen in Fig. 1-a. In contrast that of (E)MFA UL slot $\left(T_{m_{-} s l t}\right)$ consists of two parts as shown by Fig. 1-b: $T_{p k t}$ and the voting field duration $(d \Delta \tau)$ each of duration $\Delta \tau$; an additional signal pulse placed at random within the $d$ bins is sent during this period. The reader measures voting mini-slots corresponding to a data packet $S_{x}$. In case of a packet collision (i.e. multiple tags choosing the same UL slot), the slot is split subsequently into $m$ slots for collision arbitration as needed, where $m(\geq 1)$ is a variable chosen depending on the size of $d$. There are two distinct advantages of the (E)MFA idea over ISO 18000-6 Type $B$ (ISOB) standard [1] that uses a fixed binary splitting size. Firstly, an estimate of the number of contending sources is available via observation of the voting min-slots corresponding to a UL data slot; this allows choice of dynamic splitting size per slot and enhanced MAC efficiency. Secondly, even with multiple packets $(n \geq 2)$ access within a UL slot $S_{x}$ (i.e. a collision), some mini-slots will carry a singleton signal with high probability implying that the corresponding data packet(s) are guaranteed to be successfully received in the very next retransmission.

Cleary, larger $d$ implies that more chances for a mini-slot to contain a singleton signal are available, as well as that more accurate estimate of the number of contending sources is achieved. On the other hand, increasing the voting field will ultimately lead to throughput degradation as the overhead increase starts to negate any gains from the accurate estimate of collision events. The actual throughput is thus a function of 


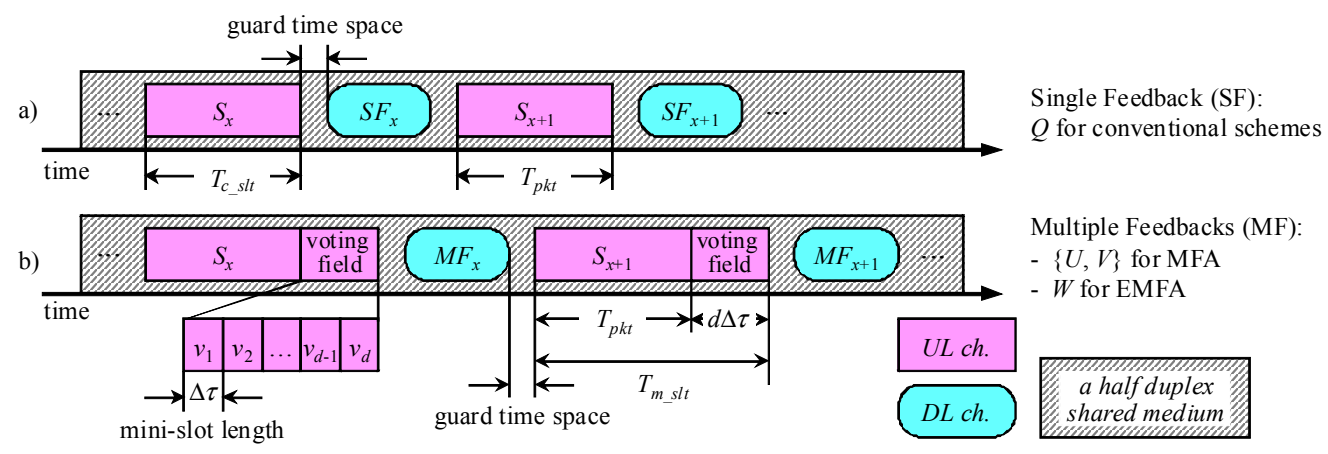

Fig. 1 The UL time slot structure for conventional schemes (a) and (E)MFA (b) in a half duplex shared medium

the ratio $\gamma_{m}=\Delta \tau / T_{p k t}$. There exist two major shortcomings in the previous MFA[3]: firstly, the extra slot time portion $(d \Delta \tau)$ was neglected in calculating the UL throughput. So it was not clear how much practical gain could be expected from the MFA. Secondly, information of a binary type feedback symbol for each $\Delta \tau$ is not sufficient for dynamic splitting size for a collided slot: a feedback for $v_{i}$ is either silent meaning no occupation of signal or not silent meaning at least one signal occupation. This issue will be discussed more specifically in the following section. In this paper, taking $d \Delta \tau$ time into consideration we develop new analytical models for MFA and EMFA. Our mathematical results are also supported by simulation results. In particular in order to better facilitate the dynamic splitting size, EMFA uses a ternary type feedback symbol $(W)$ rather than a binary one $(V)$ for $v_{i}$. We examine the value of $d$ that optimizes UL throughput for both MFA and EMFA and the achieved gain from them over ISOB, the baseline protocol.

We organize the remainder of the paper as follows. Section II illustrates the MAC protocols structure of the MFA and EMFA in depth. In chapter III, analytical models for MFA, and EMFA are derived. In section IV, the maximal throughput and gain as a function of $\gamma_{m}$ via analytical and simulation results are discussed. Finally we draw our conclusion in section $\mathrm{V}$.

\section{II. (ENHANCED) Multiple FeEdBACK AlgorithM: (E)MFA}

\section{A. $M F A$}

A slot duration $\left(T_{s l t}\right)$ in MFA consists of two parts: packet duration $T_{p k t}$, and voting field $d \Delta \tau$, where a tag that transmitted its packet to $S_{x}$ sends an additional signal pulse at random within one of the $d$ mini-slots. In MFA, then, the reader broadcasts a feedback couple $\{U, V\}$ at the end of each slot according to DMSA protocol: $U$ for the sensing result of $T_{p k t}$ is a binary feedback defined as (1) and $V$ for the sensing results of $d \Delta \tau$ is a binary $d$-tuple random variable, $\left(V_{1}, V_{2}, \ldots\right.$, $V_{d}$ ) defined as (2). Thus as long as $U=1$ the $S_{x}$ needs to be split promptly into $m$ according to the DMSA protocol as defined by (3). Then the collided packets in $S_{x}$ will be retransmitted in an orderly fashion within $m$ slots via $d$-bit sequence in $V$. Fig. 2-a illustrates an example of collision resolution process in MFA with dynamic splitting. Suppose that four packets $-p k t_{1}, p k t_{2}, p k t_{3}$, and, $p k t_{4}-$ are to be sent in slot $S_{x}$ with three mini-slot $(d=3)$; two of them $\left(p k t_{2}\right.$ and $\left.p k t_{3}\right)$ send their voting signal to $v_{1}$ and the rest ( $p k t_{1}$ and $\left.p k t_{4}\right)$ to $v_{3}$ and $v_{2}$, respectively. The corresponding feedback for $S_{x}\{U$, $V\}_{x}$ is $\{1,(1,1,1)\}$. In the next retransmission, $p k t_{2}$ and $p k t_{3}$ are supposed to compete again at $S_{x+1}$ : because the $\{U, V\}_{x+1}$ is $\{1,(1,0,1)\}$ they can be identified in the following retransmission. Slot splitting is no longer required in $S_{x+2}, S_{x+3}$, $S_{x+4}$, and $S_{x+5}$ since $U=0$. At the beginning of an inventory session the reader broadcasts the command including the size of $d$ and the exponent $q$ that sets the size of initial contention window $\left(f_{0}\right)$ to $2^{q}$, where $q=0,1,2, \ldots$ Fig. 2-b shows the logical procedure of a MFA tag in transmitting the packet. $e_{i}$ denotes the number of silent mini-slots prior to $v_{i}$ right before a non-silent mini-slot: the tag retransmitted $p k t_{2}$ to $S_{x+1}$ and sent a corresponding voting signal to $v_{3}$ sets its transmission counter $\left(t_{c}\right)$ to 2 instead of 3 after receiving feedbacks. Once an identification session starts Step $B$ and Step $C$ keep alternated in a tag until $U=0$.

$$
\begin{gathered}
U= \begin{cases}0, & \text { if Empty or Success } \\
1, & \text { otherwise, }\end{cases} \\
V_{i, x}= \begin{cases}0(\text { Empty }), \text { if } v_{i} \text { is silent } \\
1(\text { Nonempty }), & \text { if } v_{i} \text { is not silent. }\end{cases}
\end{gathered}
$$

$$
m_{x}=\sum_{i=1}^{d} V_{i, x}
$$

\section{B. Enhanced MFA}

In EMFA, a binary $d$-tuple random variable, $V$ is redefined by $W$ with a ternary $d$-tuple random variable, $\left\{W_{1}, W_{2}, \ldots\right.$, $\left.W_{d}\right\}_{x}$ for $S_{x}$ feedback as (4). The $U$ for $T_{p k t}$ in (1) is no longer used since tags can infer the result from the information in $W$ only. We assume that the reader channel sensing capability is sensitive enough to distinguish the signal multiplicity reliably even in each $v_{i}$.

$$
W_{i, x}=\left\{\begin{array}{l}
0 \text { (Empty), } \text { if no signal arrives at } v_{i}, \\
1 \text { (Success), } \text { if a single signal arrives at } v_{i}, \text { and } \\
2 \text { (Collision }), \quad \text { if more than one signals arrive at } v_{i} .
\end{array}\right.
$$

Fig. 3-a illustrates the difference how dynamic slot splitting for a collided slot $S_{x}$ is performed between MFA and EMFA. We use the same scenario of four packets voting 


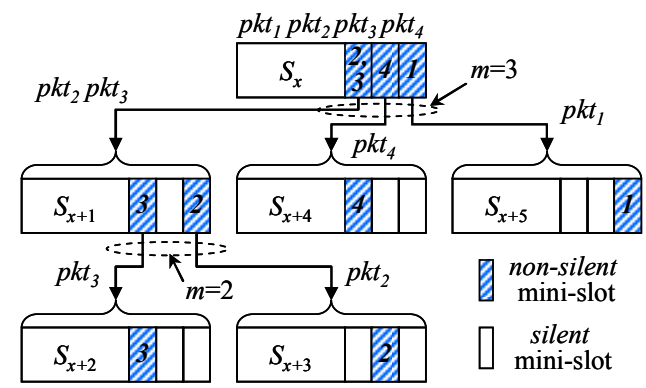

a) The collision resolution process in MFA

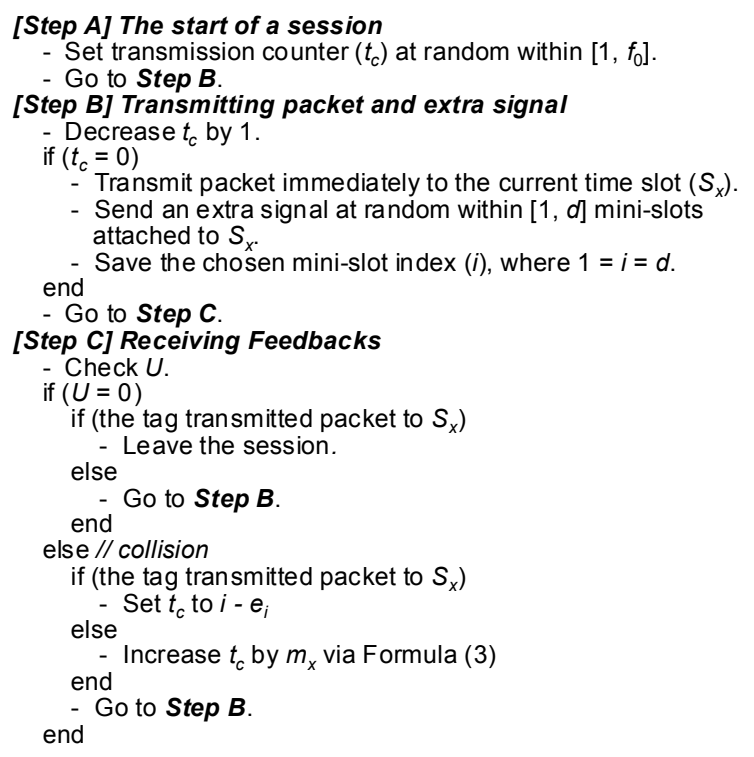

b) UL channel access process in an MFA tag Fig. 2 The functional logic flow in a MFA tag

result as the $S_{x}$ in Fig. 2 -a. Then $W_{x}$ should be $\{2,1,1\}$. It is obvious that $p k t_{2}$ and $p k t_{3}$ in MFA are destined to be collided again at their next retransmissions to $S_{x+1}$. However those in EMFA get a chance to choose one of two split slots $-S_{x+1}$ and $S_{x+2}$ - due to the fact that $v_{1}=2$. A tag that transmitted its packet to the $S_{x}$ needs to check the slot collision decision function $\left(C_{x}\right)$ as defined by (5). If $C_{x}=0$ the tag leaves the session. Otherwise $\left(C_{x}=1\right)$ the tag needs to identify its retransmission time according to the other mini-slot feedbacks in $W_{i, x}$. We define $s_{i, x}$ and $c_{i, x}$, the number of, respectively, successful and collided mini-slots up to the $v_{i}$ in $S_{x}$ to where the extra signal was sent by the tag as (6). Then the packet

TABLE I

Properties of TREe BASED RFID ANTI-COLLISION SCHEMES

\begin{tabular}{|c|c|c|c|c|}
\hline \multicolumn{2}{|c|}{$\begin{array}{c}\text { Tree Based Anti-collision } \\
\text { Schemes }\end{array}$} & \multirow{2}{*}{$\begin{array}{c}\text { Splitting } \\
\text { Size }\end{array}$} & $\begin{array}{c}\text { Degree of } \\
\text { Dynamic }\end{array}$ \\
\hline \multirow{2}{*}{ Root } & Variation & $\begin{array}{c}\text { Tag } \\
\text { estimation }\end{array}$ & & \\
\hline \multirow{3}{*}{ MSA } & default & $\begin{array}{c}m=2,3, \\
4, \ldots\end{array}$ & $\begin{array}{c}\text { session } \\
\text { variable(static) }\end{array}$ \\
\cline { 3 - 5 } & \multirow{2}{*}{ DMSA } & MFA & $m=[1, d]$ & slot variable \\
\cline { 3 - 5 } & \multirow{2}{*}{ BMSA[9] } & $\begin{array}{c}\text { EMFA } \\
\text { collision } \\
\text { rate per } \\
\text { frame }\end{array}$ & $\begin{array}{c}m=[2,2 d] \\
m=[2, k], \\
\text { where } \\
k \geq 3\end{array}$ & slot variable \\
frame variable \\
\cline { 3 - 4 }
\end{tabular}

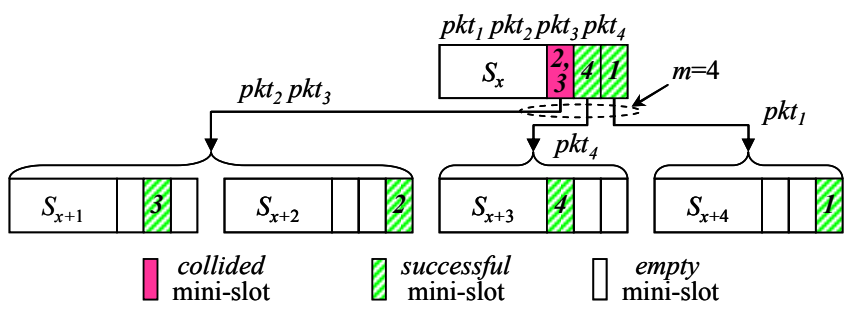

a) The collision resolution process in EMFA

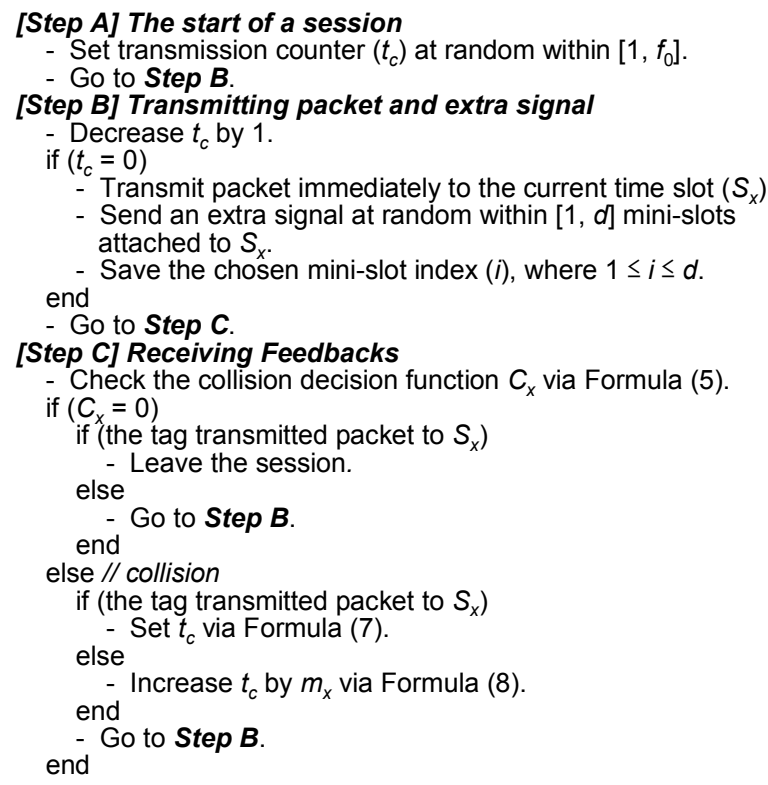

b) UL channel access process in an EMFA tag

Fig. 3 The functional logic flow in an EMFA tag

transmission counter, $t_{c}\left(W_{i, x}\right)$ for a tag that sent an extra signal to $v_{i}$ in $S_{x}$ is defined by (7) where $\operatorname{rand}[A, B]$ is the function that chooses a random number between $A$ and $B$ inclusive. Using (6) the splitting size for a collided $S_{x}\left(C_{x}=1\right)$ is given by (8). Fig. 3-b depicts the logical procedure for an EMFA tag in transmitting the packet. Once an identification session starts Step $B$ and Step $C$ keep alternated in a tag until $C_{x}=0$. Hence in (E)MFA $m_{x}$ is a slot variable, whereas $d$ is a session variable. The properties of tree based RFID anti-collision schemes are summarized in Table 1.

$$
\begin{gathered}
C_{x}=\left\{\begin{array}{l}
0, \text { if } \sum_{i=1}^{d} W_{i, x}<2 \\
1, \text { othwise }
\end{array}\right. \\
\left\{\begin{array}{l}
s_{i, x}=\sum_{a=1}^{i} W_{a, x}, \text { such that } W_{a, x}=1 \\
c_{i, x}=\sum_{a=1}^{i} W_{a, x}, \text { such that } W_{a, x}=2
\end{array}\right. \\
t_{c}\left(W_{i, x}\right)=\left\{\begin{array}{c}
s_{i, x}+2 c_{i, x}, \quad \text { if } W_{i, x}=1 \\
\operatorname{rand}\left[s_{i, x}+2 c_{i, x}-1, s_{i, x}+2 c_{i, x}\right], \quad \text { if } W_{i, x}=2
\end{array}\right. \\
m_{x}=s_{d, x}+2 c_{d, x}
\end{gathered}
$$




\section{ANALYSIS OF THE SYSTEMS}

In order to estimate the number of contending sources, consider the situation where unknown number $n(\geq 2)$ tags choose the same UL slot along with $d$ mini-slot voting signals. The distribution of the $n$ voting signals in the $d$ mini-slots is therefore multinomial as (9)

$$
M\left(b_{1}, b_{2}, \ldots, b_{d} ; n\right)=\left(\begin{array}{c}
n \\
b_{1}, \ldots, b_{d}
\end{array}\right) \prod_{i=1}^{d} p_{i}^{b_{i}}=\frac{n ! d^{-n}}{\prod_{i=1}^{d} b_{i} !}
$$

where the $d$ bins contain $b_{1}, \ldots, b_{d}$ signals respectively such that $\sum_{i=1}^{d} b_{i}=n$ and $p_{i}=1 / d$ (each mini-slot is chosen equally). The first term in the middle equation is multinomial coefficient defined by $n ! / \prod_{i=1}^{d} b_{i} !$. The number of all possible outcomes of the vector $\left(b_{1}, \ldots, b_{d}\right)$ is ${ }_{d} H_{n}\left({ }_{{ }_{d+n-l}} C_{n}\right)$, i.e., the number with repetitions of arrangements of $n$ objects into $d$ bins. Let $N(n)$ denote the number of additional UL time slots that are required to resolve an initial $n$-packet collision. Note that $N(0)=0, N(1)=0$ serve as initial conditions since there are no collisions with either 0 or 1 packet access in a slot.

\section{A. Analysis of $M F A$}

Let $X=\Sigma V_{i}$ from (2), the random variable taking values in the range $[1,2, \ldots, d]$. Then $N(2), N(3)$ can be deduced from (10), and thus $N(n)$ can be recursively computed as given by (11). For a given probability of $M(\cdot), X$ plus the additional number of slots required to resolve the subsequent collisions from $b_{1}$ to $b_{d}$ are consumed after $n$ tags cast a vote within $d$ mini-slots. Our delay derivation for MFA yields the same results as Appendix A in [3]. However because an UL slot time in MFA contains an overhead portion as illustrated in Fig. 1 -b we need to take the duration $(d \Delta \tau)$ into consideration to estimate the practical gains from (E)MFA; hence the time slot duration $\left(T_{m \_s l t}\right)$ is represented by $T_{p k t}\left(1+d \gamma_{m}\right)$.

\section{B. Analysis of EMFA}

We denote $Y=\Sigma W_{i}$ the random variable for the size of splitting slots taking the range $[2,3, \ldots, 2 d]$ for $S_{x}$ whose $C_{x}=$ 1. Then with the similar fashion with MFA $N(n)$ for EMFA can be recursively derived by (12). Therefore for a given probability of $M\left(b_{1}, \ldots, b_{d} ; n\right) M\left(\varepsilon_{1}, \ldots, \varepsilon_{\alpha} ; b_{1}\right), \ldots, M\left(\omega_{1}, \ldots\right.$, $\omega_{\alpha} ; b_{d}$ ), where $\alpha$ is 1 if $b_{i}<2$, or 2 otherwise, the number of $Y$ plus the additional number of slots to resolve subsequent collisions from $N\left(\varepsilon_{1}\right)$ to $N\left(\omega_{\alpha}\right)$ are consumed. Let us consider about initial packet loading factor $\left(\lambda_{0}\right)$ i.e., packet arrival rate in a UL time slot. Then $\lambda_{0}$ is defined by $n p^{*}$, where $n$ is the total tag pool, and $p^{*}$ is the probability that a tag in $n$ is selected by the reader for an inventory session. Since once it is selected a tag transmits its packet with probability 1 within $f_{0}$, $\lambda_{0}$ becomes $n p^{*} / f_{0}$. Then the number of packets arrival event can be represented by binomial random variable with parameters, $n$ and $p^{*} / f_{0}$. Thus we have $\bar{N}$ as (13). The first term of $\bar{N}$ means the initial frame length, and the second term is for the additionally required slots needed to serve the collided tags in the first frame. Now we define normalized UL throughput $\left(\rho_{U L}\right)$ for a CRP, which can be expressed by (14).

$$
\begin{aligned}
& N(2)=M(2,0, \cdots, 0 ; 2)\left[T_{m_{-} s l t}+N(2)+N(0)+, \cdots,+N(0)\right]+, \cdots,+ \\
& M(0,0, \cdots, 2 ; 2)\left[T_{m_{-} s l t}+N(0)+, \cdots,+N(0)+N(2)\right]+, \cdots,+ \\
& M(1,1, \cdots, 0 ; 2)\left[2 T_{m \_s l t}+N(1)+N(1)+, \cdots,+N(0)\right]+, \cdots,+ \\
& M(0, \cdots, 1,1 ; 2)\left[2 T_{m_{-} s l t}+N(0)+, \cdots,+N(1)+N(1)\right] . \\
& N(n)=M(n, 0, \cdots, 0 ; n)\left[T_{m_{-} s l t}+N(n)\right]+, \cdots,+ \\
& M(0,0, \cdots, n ; n)\left[T_{m \_s l t}+N(n)\right]+, \cdots,+ \\
& M(n-1,1, \cdots, 0 ; n)\left[2 T_{m_{-} s l t}+N(n-1)\right]+, \cdots,+ \\
& M(0, \cdots, 1, n-1 ; n)\left[2 T_{m \_s l t}+N(n-1)\right] \\
& =\sum_{\substack{0 \leq b_{i} \leq n, 1 \leq i \leq d}}^{d H_{n}}\left[M\left(b_{1}, \cdots, b_{d} ; n\right)\left\{X T_{m_{-} s l t}+\sum_{i=1}^{d} N\left(b_{i}\right)\right\}\right]
\end{aligned}
$$

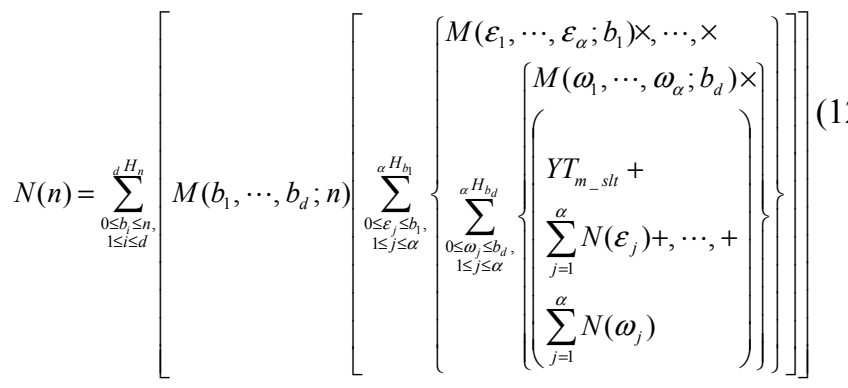

$$
\begin{aligned}
& \bar{N}=f_{0}\left\{T_{m_{-} s t}+\sum_{l=0}^{n} N(l) P(l)\right\}, \text { where } P(l)=\left(\begin{array}{l}
n \\
l
\end{array}\right)\left(\frac{p^{*}}{f_{0}}\right)^{l}\left(\frac{f_{0}-p^{*}}{f_{0}}\right)^{n-l} \\
& \rho_{U L}=\frac{\text { successful packet times }}{\text { total UL times }}=\frac{\lambda_{0}}{\bar{N}}
\end{aligned}
$$

\section{IV.PERFORMANCE EVALUATION}

We assume that the packet length is 16-bit long as in [2], and the extra signal length is 1-bit $\left(\gamma_{m}=6.25 \%\right)$ and 2-bit $\left(\gamma_{m}\right.$ $=12.5 \%$ ) long. The channel or air interface is assumed perfect, i.e. no packet losses. The lines and dots in Fig. 4 thru Fig. 7 represent analytical results and simulation results, respectively. As seen in Fig. 4 where $\gamma_{m}=6.25 \%$, a longer $d$ on one hand increases throughput over the baseline protocol, ISOB (DMSA with $m=2$ ), but on the other hand contributes progressively to increased overhead; eventually there is no gain beyond $d=4$ or 5 for MFA (Fig. 4 -a) and $d=3$ or 4 for EMFA (Fig. 4-b) within $\lambda_{0}=10$. We notably observe the low $\lambda_{0}$ regions, i.e., approximately prior to 0.5 for MFA and 0.4 for EMFA, where (E)MFA is outperformed by ISOB. This is because packets are much less likely to be collided and the presence of the voting field only increases overhead. Beyond these points where collisions increasingly prevail, (E)MFA starts to yield the gain depending on the size of $d$. If $\gamma_{m}$ gets bigger the optimal $d$ for both schemes is decreased because the increased overhead portion overwhelms the gain sooner than the shorter $\gamma_{m}$. Thus the optimal $d$ at $\gamma_{m}=12.5 \%$ is 


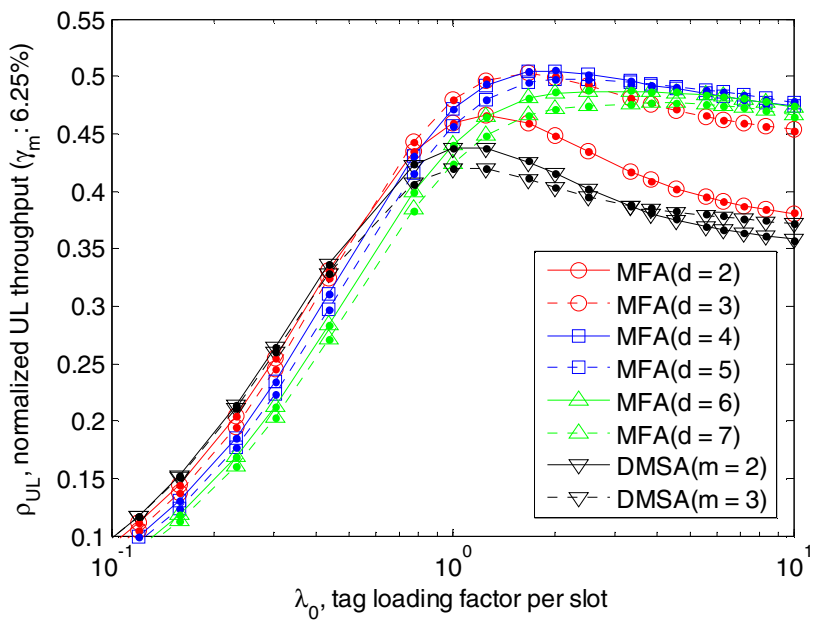

a) MFA

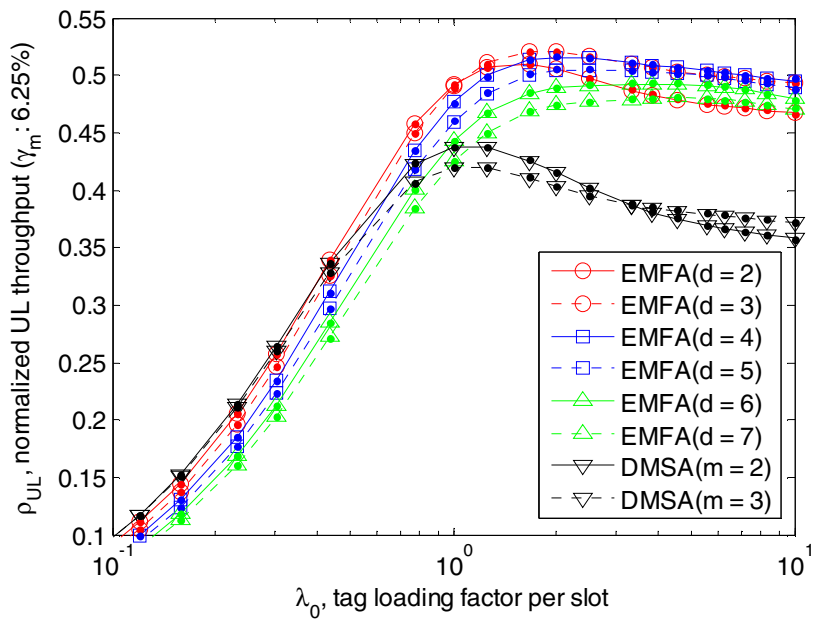

b) EMFA

Fig. 4 Performance comparison of the normalized UL throughput between MFA (a) EMFA (b) when $\gamma_{m}=6.25 \%$

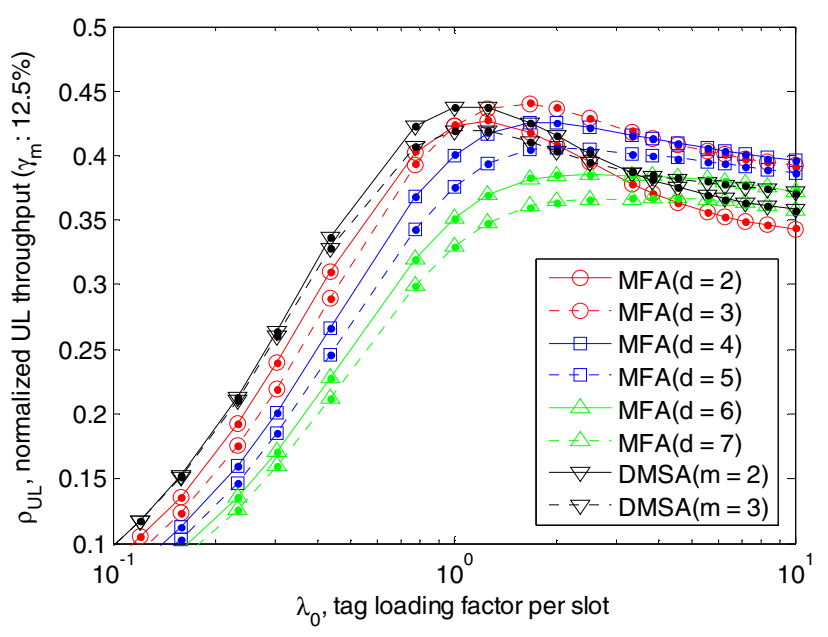

a) MFA

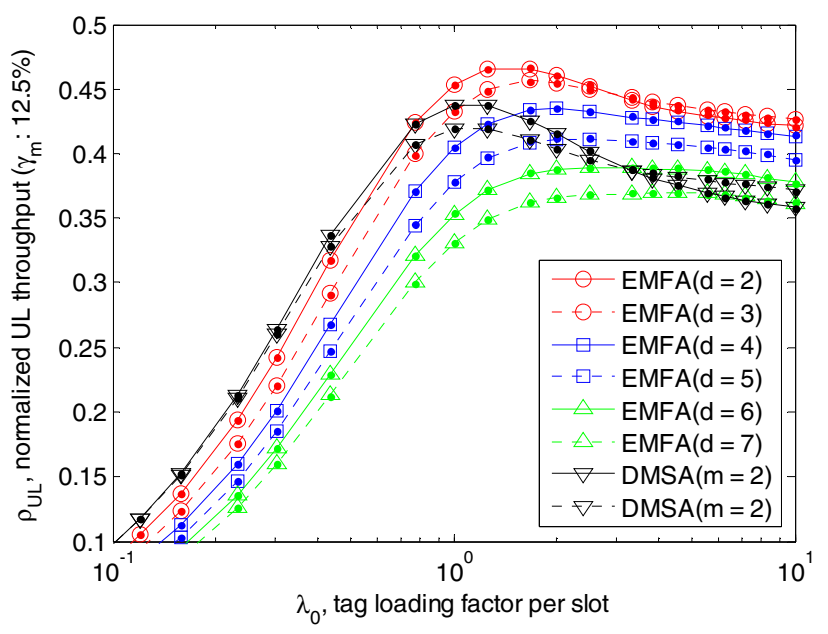

b) EMFA

Fig. 5 Performance comparison of the normalized UL throughput between MFA (a) EMFA (b) when $\gamma_{m}=12.5 \%$

decreased to 3 or 4 for MFA and to 2 or 3 for EMFA as shown by Fig. 5-a) and Fig. 5-b), respectively. A bigger $\gamma_{m}$ also makes the break-even point where (E)MFA starts to achieve gain postponed; with $d=3$ MFA starts to yield the gain beyond $\lambda_{0}=1.2$, whereas EMFA brings gain beyond $\lambda_{0}=$ 0.7 . We also observe MFA that when $\gamma_{m}=12.5 \%$ it hardly increases the maximal throughput over ISOB. However EMFA can still be higher than it. Fig. 6 shows the maximal $\rho_{U L}$ comparison with various sizes of $f_{0}$. The maximal $\rho_{U L}$ at $\gamma_{m}=6.25 \%$ for EMFA, MFA, and ISOB is, respectively, 0.52 , 0.5 , and 0.44 , and the maximal gain from MFA and EMFA over ISOB is, respectively, $31 \%$ and $36 \%$ as in Fig. 6-a. The maximal $\rho_{U L}$ at $\gamma_{m}=12.5 \%$ for EMFA, MFA, and ISOB is, respectively, $0.47,0.44$, and 0.44 , and the maximal gain from MFA and EMFA over ISOB is, respectively, $8 \%$ and $17 \%$ as shown by Fig. 6-b. Therefore the overall performance of (E)MFA outperforms that of ISOB: especially it is more robust when offered load is high. In particular it is shown that EMFA is superior to MFA because 1) EMFA achieves higher $\rho_{U L}$ through a broader range of $\lambda_{0}$ than MFA does, and 2) EMFA is less sensitive to the value of $\gamma_{m}$ than MFA since the enhanced sensing result for a mini-slot facilitates a better dynamic splitting size even with the smallest size of $d$ (2). The Fig. 7-a and 7-b illustrate how splitting size in MFA and EMFA, respectively, adapts dynamically to increased $\lambda_{0}$. The split size in (E)MFA dynamically changes as a function of $\lambda_{0}$ intensity adapting to slot based estimate, whereas it is fixed in ISOB regardless of $\lambda_{0}$. As seen in the figures the splitting size in EMFA increases more proportional to $\lambda_{0}$ than that in MFA.

\section{CONCLUSIONS}

We have introduced a novel anti-collision principle multiple feedbacks algorithm (MFA) - into RFID MAC protocols and proposed its enhanced version (EMFA). (E)MFA enables the reader to split the collided slot dynamically according to the slot based backlogged tag estimate. In order to examine the practical gain from (E)MFA we derived the optimal voting field size $(d)$ for both schemes that yields the maximal UL throughput as a function of $\gamma_{m}$, ratio of mini-slot duration $\Delta \tau$ to packet duration $T_{p k t}$. As a result with the optimal $d$ at $\gamma_{m}=6.25 \%$, MFA and EMFA achieve significant throughput gains $(31 \%$ and $36 \%$, respectively) relative to the conventional RFID spec. (ISOB) 


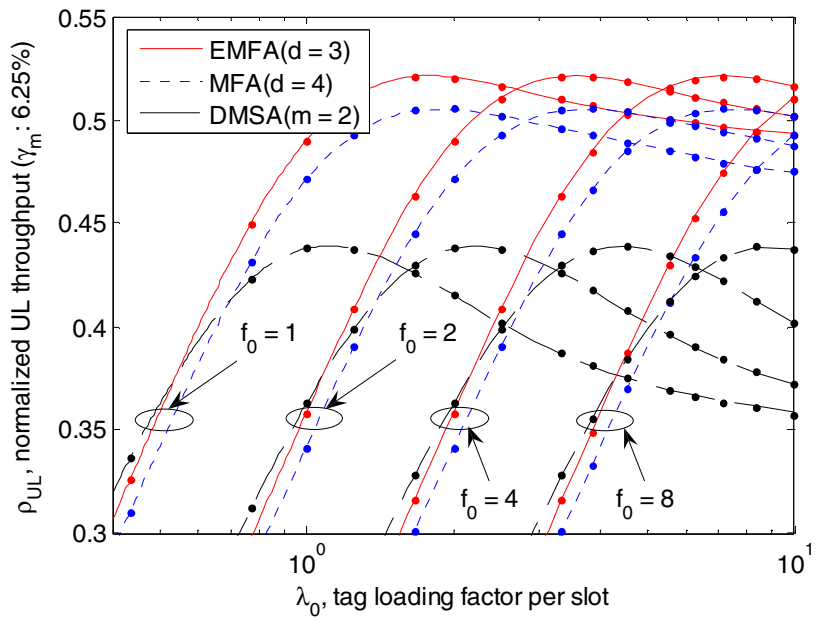

a) The maximal $\rho_{U L}$ when $\gamma_{m}=6.25 \%$

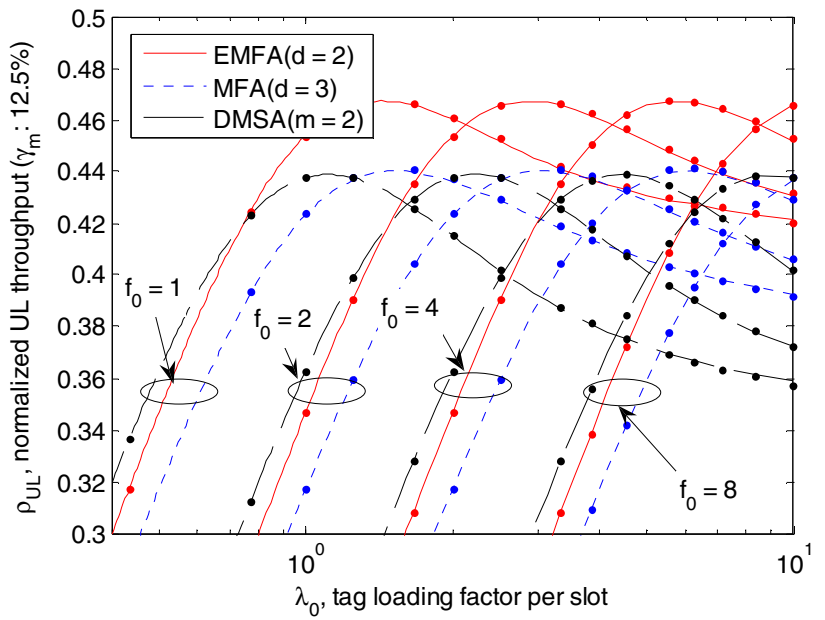

b) The maximal $\rho_{U L}$ when $\gamma_{m}=12.5 \%$

Fig. 6 The comparison of maximal $\rho_{U L}$ with various $f_{0}$ sizes in MFA and EMFA

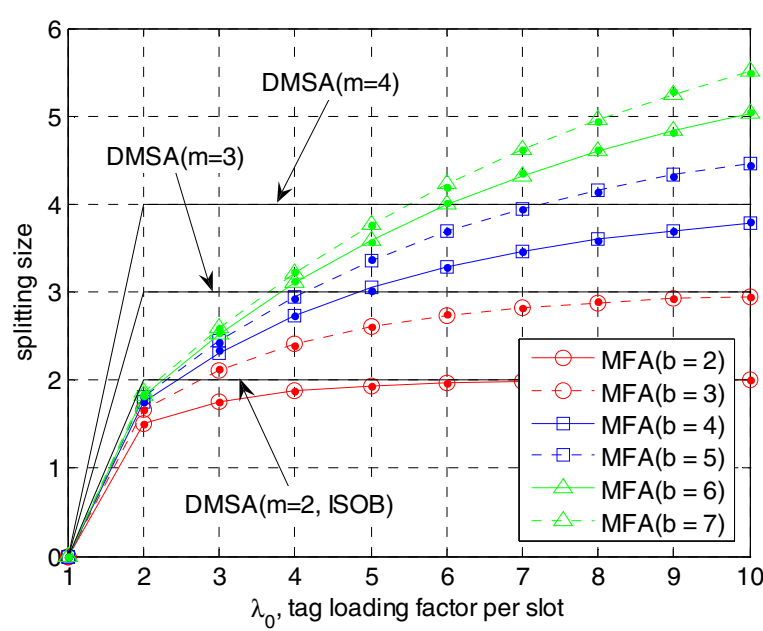

a) MFA

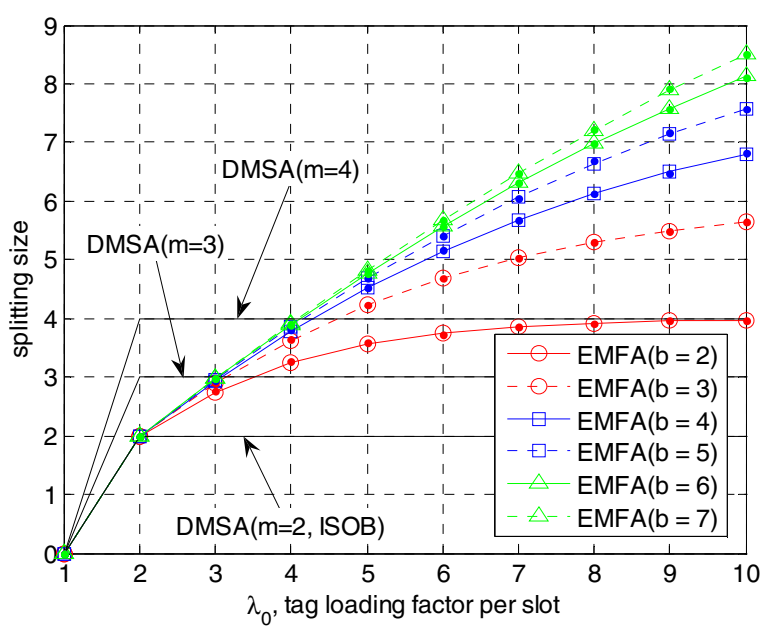

b) EMFA

Fig. 7 Dynamic splitting size in MFA (a) and EMFA (b)

[1]. Since the real size of tag pool is unknown at the start of an inventory session, the initial tag loading factor $\lambda_{0}$ can be set conservatively; (E)MFA is shown to be more robust than ISOB in this situation. The value of $\gamma_{m}$ is critical for (E)MFA performance; the bigger the value of $\gamma_{m}$ the worse the performance is. Yet EMFA is less sensitive to $\gamma_{m}$ than MFA since an enhance sensing feedback per mini-slot enables EMFA to take advantage of a better dynamic splitting size even with the smallest $d(2)$. In order to get DL gain, for a further study, (E)MFA can be run on top of breadth-firstsearch m-ary splitting algorithm (BMSA)[10], whereby DL time is greatly reduced.

\section{REFERENCES}

[1] ISO/IEC 18000-6: 2003(E), Part 6: Parameters for air interface communications at 860-960 MHz, Nov. 26, 2003.

[2] ISO/IEC 18000-6: 2005(E), Part 6C: parameters for air interface communications at $860 \mathrm{MHz}$ to $960 \mathrm{MHz}, 2005$.

[3] J-C Huang and T. Berger, "Delay Analysis of IntervalSearching Contention Resolution Algorithms", IEEE Trans. on Information Theory, Vol. IT-31, No.2 March 1985.
[4] J. I. Capetanakis, "Tree Algorithms for Packet Broadcast Channels", IEEE Trans. on Information Theory, Vol.25, No.5, Sept. 1979.

[5] J. Myung, W. Lee and J. Srivastava, “Adaptive Binary Splitting for Efficient RFID Tag Anti-collision", IEEE Comm. Letters, Vol. 10, No.3, Mar. 2006.

[6] D-K Kwon, W-J Kim, and H-N Kim, "Improvement of Anticollision Performance for the ISO 18000-6 Type B RFID system", IEICE Trans. on Communication Vol.E90-B, No.8, Aug. 2007.

[7] C. Floerkemeier and M. Wille, "Comparison of Transmission Schemes for Framed Aloha based RFID Protocols", IEEE SAINTW 2006.

[8] B. Zhen, M. Kobayashi and M. Shimizu, "Framed ALOHA for Multiple RFID Objects Identification", IEICE Trans. on Communications, vol. E88-B(3), Mar 2005.

[9] Y-C Ko, S. Roy, J. R. Smith, H-W Lee, and C-H Cho, "An Enhanced RFID Multiple Access Protocol for Fast Inventory", IEEE Globecom, Washington D.C., 2007.

[10] Y-C Ko, S. Roy, J. R. Smith, H-W Lee, and C-H Cho, "RFID MAC Performance Evaluation Based on ISO/IEC 18000-6 Type C", IEEE Comm. Letters, Vol. 12, No. 6, June 2008. 